Documentos PRESEEA de investigación

Guía PRESEEA para la investigación lingüística

Francisco Moreno Fernández (ed.)
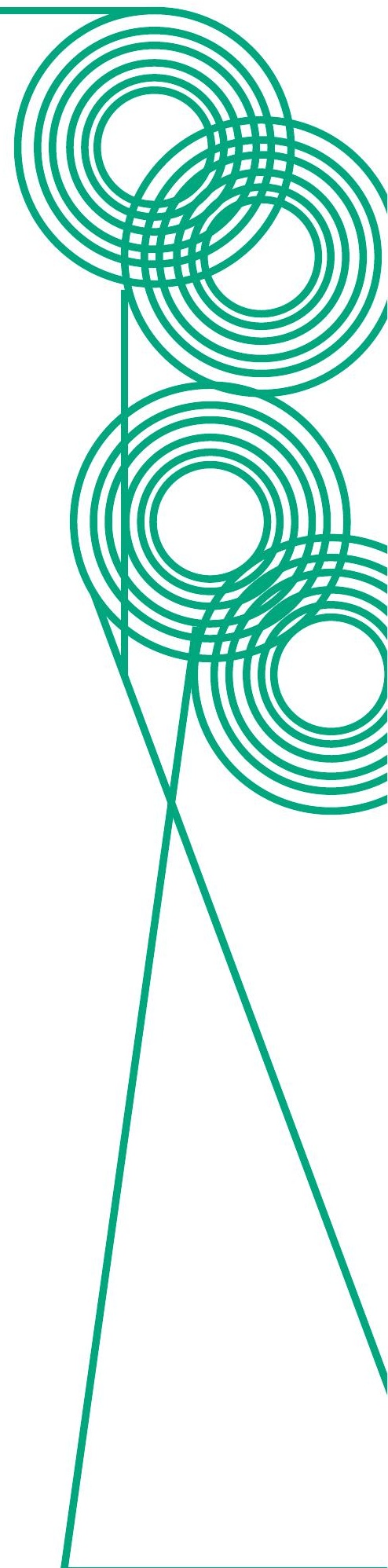

DOCUMENTOS 
Reservados todos los derechos. Queda prohibida, salvo excepción prevista en la ley, cualquier forma de reproducción, distribución, comunicación pública y transformación de esta obra sin contar con la autorización de los titulares de propiedad intelectual. La infracción de los derechos mencionados puede ser constitutiva de delito contra la propiedad intelectual (arts. 270 y sigs., Código Penal). El Centro Español de Derechos Reprográficos (www.cedro.org) vela por el respeto de los citados derechos.

Esta publicación ha sido financiada por el Grupo de investigación Lingüística de la variación espacial y social (LIVARES) y por el Proyecto para el estudio sociolingüístico del español de España y Amémica (PRESEEA).

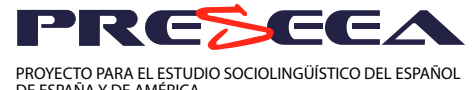
PROYECTO PARA EL ESTUDIO SOCIOLINGÜISTICO DEL ESPAÑOL
DEESPANNAY DE AMERICA
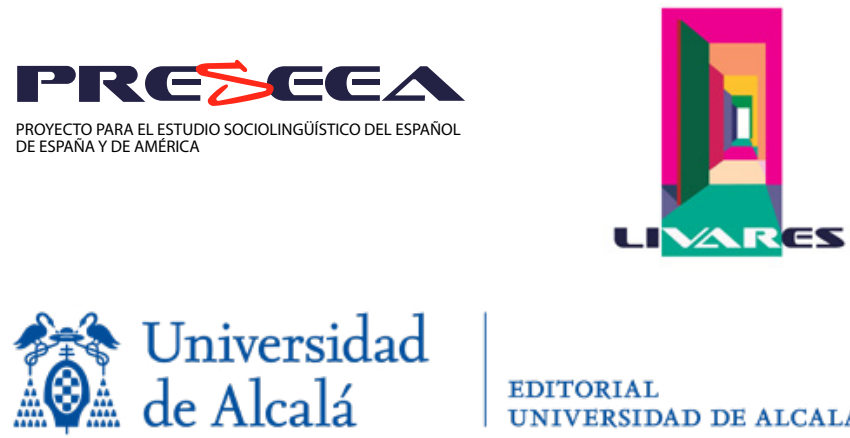

EDITORIAL

UNIVERSIDAD DE ALCALÁ

(C) Francisco Moreno Fernández

Editan: Proyecto para el estudio sociolingüístico del español de España y

América (PRESEEA)

Colegio San José de Caracciolos

C/Trinidad, $5 \cdot 28801$ Alcalá de Henares (Madrid, España)

Web: https://preseea.linguas.net/

Editorial Universidad de Alcalá, 2021

Plaza de San Diego, s/n.ํ • 28801, Alcalá de Henares (España)

Web: uah.es 


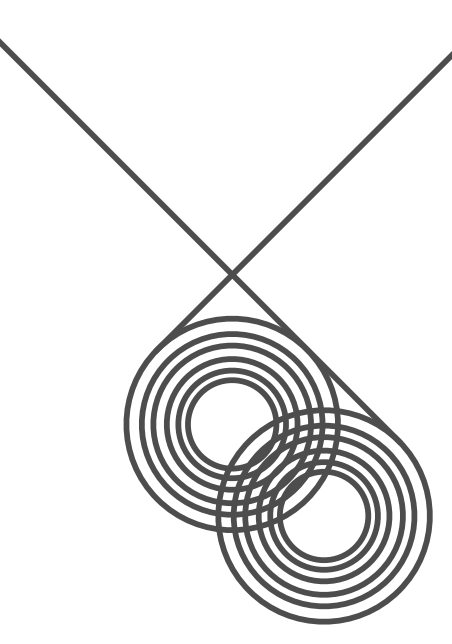

\section{Guía PRESEEA para la investigación lingüística}

Francisco Moreno Fernández (ed.) (Universidad de Alcalá -España) francisco.moreno@uah.es

\section{PUREDEE}

PROYECTO PARA EL ESTUDIO SOCIOLINGÜISTICO DEL ESPAÑOL DE ESPAÑA Y DE AMÉRICA

2021
DOCUMENTOS

PRESEEA DE TRABAJO 


\section{Índice}

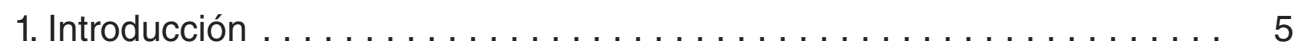

2. La investigación lingüística en PRESEEA: objetivos generales y objetos

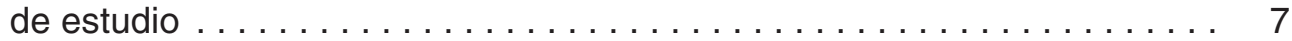

3. Constitución de los grupos PRESEEA de investigación $\ldots \ldots \ldots \ldots 9$

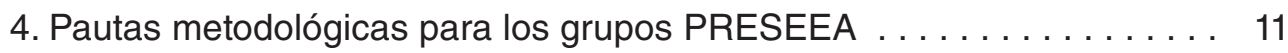

5. Confidencialidad de los datos, garantía de anonimato y ética . . . . . . 14

6. Presentación, publicación y difusión de las investigaciones. . . . . . . . 16

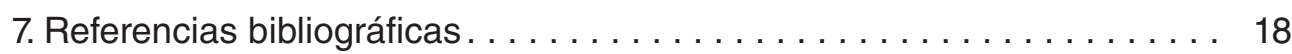

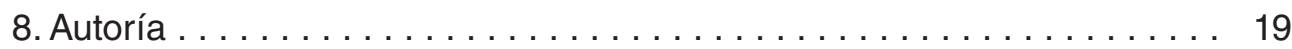




\section{Introducción}

L

as bases metodológicas del proyecto PRESEEA (Moreno Fernández 1996) establecían que la finalidad del proyecto es coordinar investigaciones sociolingüísticas de Iberoamérica y de la península ibérica para facilitar la comparabilidad de los estudios y el intercambio de información básica. Entre el origen del proyecto y el fin de su primera etapa, en diciembre de 2010, el objetivo prioritario fue la constitución de un corpus de lengua hablada que incluyera muestras representativas de las comunidades de habla investigadas, teniendo en cuenta las variables sociales más claramente correlacionadas con la conducta lingüística de los hablantes. De este modo, PRESEEA aspiraba a poner en manos de los investigadores unos materiales comparables, de origen contrastado, bien transcritos, en las mejores condiciones técnicas y de la forma más eficaz posible. La consecución de estos objetivos generales ha permitido pensar en nuevas etapas para las tareas comunes de investigación dentro del proyecto.

El mismo documento de bases metodológicas aludía a la necesidad del análisis e interpretación de los materiales lingüísticos reunidos dentro del proyecto y señalaba la libertad de que disfrutaban los investigadores vinculados a PRESEEA a la hora de afrontar esas tareas. Esa libertad se ha ejercido desde el inicio del proyecto por parte de numerosos investigadores, que han planificado sus estudios de los materiales de PRESEEA según sus particulares y siempre respetables intereses, como lo demuestran los diversos libros, artículos y tesis que se han presentado y editado. Así lo demuestran también los análisis emprendidos por parte de varios grupos de investigación, que han unido sus esfuerzos para conseguir financiación.

Ahora bien, a la hora de dar continuidad a un proyecto coordinado de esta naturaleza, la asamblea constituida por representantes de todos los equipos de la red consideró imprescindible proceder al análisis de los materiales también de una forma coordinada, sin perjuicio de que puedan realizarse otros trabajos, a partir de los mismos materiales, con finalidades más particulares o personales. Las sinergias emanadas de PRESEEA se aprovechan para proponer investigaciones ambiciosas sobre la realidad lingüística y social de la lengua española. 
Con el fin de que esas investigaciones pudieran reconocerse como parte del proyecto PRESEEA y de que contaran con unas señas de identidad que las singularizaran en el panorama de la investigación internacional, los equipos miembros de PRESEEA decidieron seguir unas mínimas pautas comunes que quedaran reflejadas en una guía o documento de referencia para la investigación coordinada.

El objetivo de esta guía para la investigación lingüística es fijar unos criterios generales, unos procedimientos comunes, unas pautas metodológicas, para proceder al análisis de los materiales de lengua hablada reunidos en PRESEEA. La intención es que, más allá de los objetos de estudios analizados y de las perspectivas teóricas que puedan adoptarse, existan unos patrones de investigación que hagan reconocibles los análisis como vinculados al proyecto PRESEEA y derivados de él. De esta forma, no solamente se pueden abordar estudios más ambiciosos, por la participación de un mayor número de investigadores, sino que se facilita la obtención de recursos internos y externos, así como una mayor financiación y difusión para los trabajos que se realicen.

Esta "Guía PRESEEA para la investigación lingüística" ofrece unos criterios y pautas generales sobre la constitución, la dinámica y la metodología de los grupos de investigación constituidos 


\section{La investigación lingüística en PRESEEA: objetivos generales y objetos de estudio}

L

as investigaciones lingüísticas originadas y coordinadas dentro del proyecto PRESEEA han de caracterizarse por su alto nivel de calidad y por ajustarse a los estándares de calidad más exigentes en el panorama de la investigación internacional. Los trabajos de PRESEEA, por la cantidad y calidad de los materiales a partir de los cuales se elaboran, han de ser referencia obligada en el estudio de la lengua española y ofrecer las máximas garantías posibles de fiabilidad y rigor en el manejo de los datos.

Los objetos de estudio de las investigaciones vinculadas a PRESEEA podrán ser cualesquiera que los equipos miembros decidan. No obstante, la red concede una mayor importancia a los siguientes fines:

a. conocer las principales líneas de evolución de la lengua española, en cualquiera de sus niveles;

b. analizar el desarrollo de la variación geolingüística y sociolingüística en el conjunto del dominio hispanohablante;

c. y hacer aportes a la teoría general de la variación y del cambio lingüístico.

Como puede apreciarse en la formulación de estas prioridades de investigación, los trabajos de PRESEEA no se vinculan a un marco teórico concreto; antes bien, permiten una aproximación desde perspectivas teóricas muy diversas, desde las más sociológicas a las más cognitivistas, desde las más universalistas a las más contextualizadas o desde la más centradas en la competencia abstracta a las más basadas en el uso. Es evidente que el trabajo a partir de un corpus favorece las aproximaciones de base inductiva y que siguen los modelos habituales de análisis basados en corpus, pero esto no es óbice para la práctica de investigaciones de perfil deductivista, que entiendan los datos como banco 
de pruebas para la comprobación de hipótesis. Del mismo modo, los materiales de PRESEEA son idóneos para la aplicación de técnicas cuantitativas de análisis, pero ello no ha de ser un obstáculo ni para la práctica de las especulaciones ni mucho menos para el desarrollo de análisis cualitativos, que en todo caso se entienden como imprescindibles para la consecución de una cuantificación adecuada.

Como primeros objetos de estudio, con prioridad para la constitución de grupos de investigación, PRESEEA propuso los siguientes:

1. Nivel fónico. Análisis de -s en posición implosiva y análisis de $-d$ - intervocálica.

2. Nivel gramatical. Análisis de usos de haber.

3. Nivel discursivo. Análisis de la atenuación.

Esta primera relación de objetos de estudio prioritarios, además de iniciar los trabajos internacionales coordinados ha servido de primera experiencia para abordar otros temas de investigación y ha permitido ofrecer, en un periodo de tiempo relativamente corto -para la complejidad de este tipo de investigaciones-, resultados que se presentan a la comunidad científica internacional con el distintivo del proyecto PRESEEA. 


\section{Constitución de los grupos PRESEEA de investigación}

urante la fase de recolección de los materiales que constituyen el corpus de lengua hablada PRESEEA, los "equipos" que llevaron a cabo las tareas de investigación se identificaron mediante el acrónimo del proyecto, la ciudad y el código del país correspondiente: PRESEEA - MONTERREY - MX. Una vez constituido el corpus, las tareas de análisis de los materiales de PRESEEA son realizadas por "grupos" de investigación que se identifican mediante la fórmula "PRESEEA_objeto de análisis" (p.e. PRESEEA_S_IMPLOSIVA; PRESEEA_HABER; PRESEEA_ATENUACIÓN). Estos grupos cuentan con un reconocimiento oficial y formal dentro del proyecto y figuran como tales en sus medios de difusión.

Los grupos PRESEEA están formados por investigadores de diversos equipos y países; es decir, son siempre grupos de composición internacional. Dentro de cada grupo podrán cumplirse distintas funciones, que quedan relacionadas y descritas seguidamente.

1. Coordinadores. Cada grupo PRESEEA cuenta con, al menos, dos coordinadores, preferentemente de países diferentes. La designación de los coordinadores se hace por asentimiento de la mayoría de los equipos, teniendo en cuenta las alternativas posibles, las sugerencias de los miembros, los intereses del proyecto general y las posibilidades de éxito de los trabajos de investigación.

2. Investigadores. Pueden ser investigadores de un grupo todos aquellos expertos que lo deseen. Los investigadores pueden ser miembros de PRESEEA o invitados externos del proyecto que puedan contribuir, con su conocimiento y trabajo, al éxito de la investigación.

Los grupos de investigación pueden contar con la colaboración de especialistas que cumplan funciones como las que se describen a continuación: 
3. Consultores. Son especialistas que, siendo o no miembros de PRESEEA y sin formar parte permanente del grupo, pueden prestarle asesoramiento, auxilio o información en tareas específicas.

4. Evaluadores externos. Los grupos de investigación podrán remitir sus trabajos, parciales o completos, a evaluadores externos con capacidad de informar o dictaminar sobre los aspectos consultados.

Cuando se recurre a estas figuras externas, se debe dar a conocer quiénes han cumplido tales funciones y sobre qué aspectos, como elemento de juicio y garantía de la investigación. Todos los integrantes y participantes en un grupo PRESEEA se identifican como tales cuando publican datos, análisis o información generados en el seno del grupo y como consecuencia de sus trabajos.

La adscripción a los grupos es siempre voluntaria y la función dentro de cada grupo viene determinada por los coordinadores. 


\section{Pautas metodológicas para los grupos PRESEEA}

$S$ investigaciones de los grupos de PRESEEA, los trabajos coordinados originados en el proyecto se ajustan a unos criterios y pautas generales que maximizan las ventajas de participar en un proyecto internacional y que sirven de señas de identidad a las investigaciones elaboradas a partir de unos materiales comunes.

Las pautas metodológicas propuestas para su general seguimiento, más allá del objeto de estudio analizado, son las que se exponen a continuación:

a. El fundamento de los trabajos de PRESEEA es el aprovechamiento del corpus de lengua hablada, reunido para su posterior análisis. Así pues, todos los grupos de PRESEEA deben utilizar los materiales del corpus PRESEEA como fuente primaria de datos para sus análisis y ejemplos, sin perjuicio de que pudieran utilizarse otros datos cuando fuera necesario.

b. Los objetivos específicos de los análisis y las pautas de trabajo de los grupos se establecen por los respectivos coordinadores, que reciben sugerencias de los investigadores miembros de su grupo.

c. La cita de cualquier fragmento del corpus PRESEEA, por breve que sea, debe acompañarse de los códigos que permitan su fácil localización: equipo e informante (p.e.: PRESEEA_MÁLAGA_H11_999). Los códigos correspondientes al perfil de los informantes, tal y como se especifica en la página electrónica del proyecto, son los siguientes: 
PRIMERA POSICIÓN

$\mathrm{H}=\mathrm{HOMBRE}$

$\mathrm{M}=\mathrm{MUJER}$

SEGUNDA POSICIÓN

1=GENERACIÓN 1 (20-34)

2=GENERACIÓN 2 (35-54)

3=GENERACIÓN 3 (>55)

\section{TERCERA POSICIÓN}

1=NIVEL DE ESTUDIOS 1 (PRIMARIOS)

2=NIVEL DE ESTUDIOS 2 (MEDIOS)

3=NIVEL DE ESTUDIOS 3 (SUPERIORES)

A la identificación general de cada cita referida al corpus, podrá añadirse su localización específica en el texto correspondiente, con referencia a la publicación en que aparezca (impresa o digital), cuando sea posible.

d. Todos los equipos PRESEEA (de todas las ciudades) están obligados a proporcionar a los grupos de investigación del proyecto los materiales que estos requieran para poder realizar sus análisis. De este modo, si un equipo dispusiera de materiales originados en PRESEEA y que, por el motivo que fuera, no hubieran sido publicados, estaría en la obligación de remitirlos a los coordinadores de los grupos que los solicitaran para realizar sus análisis (p.e. archivos de audio de mayor tamaño, materiales inéditos de determinados informantes, entrevistas complementarias no publicadas).

e. Los análisis lingüísticos de PRESEEA tienen en el contraste de usos de variedades diferentes una de sus principales características. Son habituales, pues, las comparaciones de datos de diversa procedencia dialectal o sociolectal (Tagliamonte 2013; Moreno Fernández 2019). Las variables geográficas, sociales y situacionales son exploradas como posibles variables explicativas de los fenómenos analizados, en un intento de obtener el máximo aprovechamiento de los materiales del corpus general. La no pertinencia de las variables de esta naturaleza es uno 
de los resultados posibles de los análisis y se considera igualmente significativa.

f. Las investigaciones lingüísticas de PRESEEA incorporan, en la medida de lo posible y de acuerdo con la naturaleza de sus datos y el objeto de estudio, técnicas de análisis cuantitativas. La aplicación de la cuantificación se fundamenta en análisis cualitativos apropiados y no impide el manejo de otro tipo de pruebas. En la aplicación de técnicas estadísticas, se intenta utilizar las más adecuadas a cada caso, paramétricas o no paramétricas, sean de una complejidad mayor o menor. Asimismo, se procura no aplicar de modo exclusivo pruebas de naturaleza meramente descriptiva. En este sentido, el manejo de pruebas estadísticas de inferencias se entiende como un valioso instrumento para el enriquecimiento de los análisis. En todo caso, los resultados de las pruebas estadísticas deben acompañarse de los índices de significación y fiabilidad apropiados, cuando las pruebas permitan su obtención.

g. Las muestras y ejemplos que se utilizan en los análisis de PRESEEA deben presentarse suficientemente contextualizados, tanto en lo que afecta a su origen en la enunciación, como en lo que afecta a su contexto comunicativo y lingüístico inmediato. 


\section{Confidencialidad de los datos, garantía de anonimato y ética}

n proyecto como PRESEEA debe prestar una especial atención a las delicadas cuestiones relacionadas con la utilización de grabaciones y transcripciones que contienen informaciones personales relativas a los informantes. Estas cuestiones suelen complicarse por la existencia de legislaciones diferentes según los países a los que pertenecen los distintos equipos participantes en el proyecto. El tratamiento de la confidencialidad de los datos personales y la garantía del anonimato afecta a los equipos e investigadores en distintos niveles:

- el equipo recolector de la grabación y responsable de la transcripción;

- otros equipos participantes en PRESEEA (considerados de forma individual);

- el conjunto de los equipos participantes en PRESEEA;

- el conjunto de la comunidad investigadora, que implica tanto la posibilidad de explotación abierta de los materiales recogidos, como su cesión a otros proyectos.

Los equipos responsables de la recolección de las grabaciones y de la transcripción de sus respectivos materiales deben conocer la legislación de su país y ajustarse a ella. En algunos países, como España, es habitual pedir a los entrevistados que firmen un documento de conformidad y darles la garantía de que sus datos personales no estarán a disposición de nadie ajeno al equipo investigador. La difusión de las transcripciones se hace habitualmente mediante la sustitución sistemática y congruente de todos los nombres de personas o de lugares que aparezcan en la entrevista y que puedan permitir la identificación de los hablantes.

Las transcripciones realizadas de este modo ofrecen garantías suficientes para su uso y estudio por parte de otros equipos participantes o colaboradores de PRESEEA, incluyendo la integración en el corpus general PRESEEA y su posible cesión a otros proyectos. Siendo así, corresponde a cada equipo recolector 
la responsabilidad de garantizar que los materiales que se ceden al conjunto de PRESEEA o a algún otro equipo cumplen los requisitos exigidos por la legislación existente en el país de origen. En el caso de que un equipo responsable ceda materiales a otros equipos o a miembros de otros equipos en su transcripción original, deber exigir la firma de un acuerdo de confidencialidad equivalente al suscrito por los miembros del equipo responsable.

En el caso de las grabaciones (archivos de sonido), el procedimiento más extendido para garantizar el anonimato de los informantes es la sustitución de los nombres de personas o lugares por un pitido, que debe tener la misma duración que el nombre pronunciado, para asegurar el buen funcionamiento de la alineación entre transcripción y grabación. Esta es la forma en que debe procederse para un uso abierto y generalizado de las grabaciones. Mientras el proceso de modificación de las grabaciones no se haya llevado a cabo por parte de los equipos responsables, los equipos o investigadores colaboradores debe firmar un compromiso de confidencialidad garantizando el adecuado uso de las muestras sonoras. Para el cumplimiento de este compromiso puede recurrirse al simple control de que en el fragmento seleccionado no aparece ninguna mención personal que debiera ser eliminada.

Como aval de la adecuación ética de los procedimientos y materiales reunidos y analizados en PRESEEA, el proyecto general cuenta con la certificación del Comité de Ética de Investigación de la Universidad de Alcalá (España) (Código: $\mathrm{CEI} / \mathrm{HU} / 2015 / 20)$. Este certificado puede presentarse y mencionarse por parte de los equipos de PRESEEA que aborden tareas de investigación dentro del marco general del proyecto. 


\section{Presentación, publicación y difusión de las investigaciones}

L

os grupos PRESEEA de investigación lingüística realizan su trabajo de modo que puede ser seguido y aprovechado, en la mayor medida posible, por los miembros de la red general (Moreno Fernández y Cestero 2020). Esto compromete a todos los grupos, especialmente a sus coordinadores, a ofrecer una información tan completa y actualizada como sea posible sobre el trabajo de investigación y su evolución, a través de los medios que el proyecto disponga a tal efecto.

Cada grupo de investigación pone a disposición de todos los miembros de PRESEEA, principalmente a través de la página general o de la lista de correo electrónico, los siguientes materiales:

a. Bibliografía especializada sobre su objeto de estudio específico, con los enlaces correspondientes cuando el material bibliográfico esté en Internet.

b. Los documentos o recursos que esté manejando el grupo para el desarrollo de sus análisis: relación de variables analizadas, vocabularios especializados, textos de lectura común, resultados preliminares, etc.

c. Enlaces referidos a las herramientas de análisis manejadas por el grupo: p.e. aplicaciones de estadística, programas para el análisis de la voz, páginas con repertorios bibliográficos, etc.

La página electrónica de PRESEEA sirve de apoyo a los grupos de investigación y como medio de comunicación entre todos los miembros de la red PRESEEA, permitiendo el intercambio de información y de materiales. Asimismo, en la página electrónica del proyecto se publican los borradores, las redacciones preliminares y las primeras versiones de los trabajos de investigación, sin perjuicio de que las versiones más elaboradas puedan remitirse a otras editoriales y revistas. 
Asimismo, la Universidad de Alcalá se ha hecho responsable de la publicación de la serie "Documentos PRESEEA de investigación" que incluye guías de investigación, documentos de trabajo e informes monográficos relacionados con el proyecto. De este modo se ha creado un fondo de textos que permite discutir y debatir sobre las investigaciones en curso, además de dar a conocer las líneas de trabajo del proyecto general, de forma similar a como se hace en otros ámbitos de investigación. 


\section{Referencias bibliográficas}

Moreno Fernández, F. 1996. "Metodología del 'Proyecto para el estudio sociolingüístico del Español de España y de América' (PRESEEA),' Lingüística, 8: 257-287.

Moreno Fernández, F. 2019. "Macroregional sociolinguistics: Uses and preferences on null direct objects in Spanish", Journal of Linguistic Geography, 7-1: 46-60. DOI: https://doi. org/10.1017/jlg.2019.5.

Moreno Fernández, F. y A. M. Cestero. 2020. “El proyecto PRESEEA: desarrollos analíticos." En Á. Gallego y F. Roca Urgell (eds.), Dialectología digital del español, Santiago de Compostela, Universidad de Santiago de Compostela. Verba. Monográfico 80.

Tagliamonte, Sali. 2013. "Comparative Sociolinguistics". En J. K. Chambers y N. Schilling (eds.), The Handbook of Language Variation and Change, Oxford, Wiley: 729-763. 


\section{Autoría}

Este documento, difundido desde marzo de 2011, se elaboró por acuerdo de la asamblea general de miembros de PRESEEA, reunida en Comillas (España) en septiembre de 2010. Para la redacción de este documento, se contó con informes y propuestas aportados por diversos investigadores del proyecto. Han participado en la redacción de este documento los siguientes investigadores: Paola Bentivoglio, Laura Camargo, Ana María Cestero, José Ramón Gómez Molina, Irania Malaver, Pedro Martín Butragueño, Francisco Moreno Fernández, Luis Ortiz, Lidia Rodríguez Alfano, Yolanda Rodríguez Cadena, Guillermo Rojo, José Antonio Samper, Carmen Silva, Victoria Vázquez, Juan Villena. La experiencia acumulada por todos los equipos de PRESEEA ha sido esencial para la preparación de esta guía. La actualización y edición de la versión publicada en 2021 ha estado a cargo de Francisco Moreno Fernández. 